\title{
Burden and pattern of acute diarrhea in Thai children under 5 years of age: A 5-year descriptive analysis based on Thailand National Health Coverage (NHC) data
}

\author{
Busara Charoenwat ( $\boldsymbol{\sim}$ busarcha@kku.ac.th ) \\ Khon Kaen University \\ Kunanya Suwannaying \\ Khon Kaen University \\ Watuhatai Paibool \\ Khon Kaen University \\ Napat Laoaroon \\ Khon Kaen University \\ Sumitr Sutra \\ Khon Kaen University \\ Kaewjai Thepsuthammarat \\ Khon Kaen University
}

\section{Research Article}

Keywords: acute diarrhea, children under 5 years of age, children

Posted Date: February 4th, 2022

DOI: https://doi.org/10.21203/rs.3.rs-1267390/v1

License: (c) (i) This work is licensed under a Creative Commons Attribution 4.0 International License.

Read Full License 


\section{Abstract}

Background: The incidence of acute diarrhea in Thai children under five years of age has increased over the last three decades. Even though mortality has significantly declined, the burden and cost of medical treatment are still high. Our objectives are to describe the burden and pattern of acute diarrhea cases that required admissions by Thai children under five years of age from 2015 to 2019.

Methods: Data regarding the admission of acute diarrhea cases of Thai children with Thailand National Health Coverage (NHC) under five years of age from 2015 to 2019, recorded as International Statistical Classification of Diseases and Related Health Problems, tenth Revision, Thai Modification (ICD-10-TM), were analyzed.

Results: The incidence trend of yearly acute diarrhea in children 0-5 years of age slightly decreased over approximately the last decade (124,403 cases in 2010 to 102,941 cases from 2015 to 2019), along with the high cost of performing the medical treatment of approximately three billion baths per year. Diarrhearelated mortality had a low, constant rate of 0.95 per 100,000 population per year (range: 0.81-1.14 per 100,000 population per year). Three-fourths of the mortality rate was observed in children under 2 years of age and a regional distribution of the causative agent was also observed in Cholera, Typhoid, and Amoebiasis cases. Seasonal variations demonstrated consistency with similar patterns during the cold and rainy seasons throughout the 5-year period. A08 and A09 were the two most common causes of diarrheal diseases.

Conclusions: The burden, incidence and mortality rate of acute diarrhea in Thai children under five years of age were lower than those in the past decades. A similar seasonal outbreak of acute diarrhea was seen during each examined year. The causative agent was not significant and was mainly unspecific.

Trial registration: number TCTR20220117002, date of registration 17/01/2022

\section{Background}

Diarrheal disease, which is defined as a decrease in the consistency of stool, loose stool and/or passing three or more stools in 24 hours ${ }^{1,2}$, remains the second largest cause of mortality in children, after pneumonia, globally, especially in developing countries. ${ }^{3}$ The World Health Organization (WHO) and the United Nations Children's Fund (UNICEF) estimated that there are approximately 2 billion diarrheal episodes per year and approximately 1.9 million deaths of children under 5 years of age each year. For diarrhea-related mortality, three-quarters of children are in African and Southeast Asian territories. ${ }^{3,4} \mathrm{~A}$ study by Sutra et al. ${ }^{4}$ in the last decade revealed the burden of diarrheal diseases and concluded that the incidence of diarrhea was higher than previously estimated while the mortality rate was lower. The Global Burden of Diseases, Injuries, and Risk factor Study 2015 (GBD 2015) ${ }^{5}$ estimated that diarrheal disease was a crucial cause of mortality in all generations, particularly in children under 5 years of age $(499,000$ deaths, $95 \%$ UI 447,000-558,000). However, diarrheal-related mortality decreased by $20.8 \%$ (95\% UI 15.4- 
26.1) from 2005 to 2015. The WHO has aimed to reduce diarrhea-related deaths in the under 5 years old population to below $1: 1,000$ live births by $2025{ }^{6,7}$ For our country, Thai public health policy targets reducing the burden of diarrhea in children under 5 years old and reducing diarrheal-related death to zero. In 2017, the Bureau of Epidemiology of the Ministry of Public Health of Thailand revealed that the incidence of acute diarrhea in children in the first five years of life remained constant from 2015 to 2017, with approximately 1,854 cases per 100,000 population, while diarrheal-related mortality rate dramatically declined. ${ }^{4}$ Over the past 30 years, the improvement of government health facilities services, socioeconomic status, sanitation, treated water supplies, personal hygiene, and oral rehydration salts (ORS), along with the promotion of exclusive breastfeeding, and, lastly, the rotavirus vaccine, were attributed to the improvement of diarrhea-related mortality outcomes in developing countries, and decreased diseases-related fatality in industrialized territories. ${ }^{3,5,8-10}$ In Thailand, universal health coverage was realized in 2002. ${ }^{11}$ In 2010, the three major health schemes were the Universal Coverage Scheme (UC), the Civil Servant Medical Benefit Scheme (CSMBS) and the Social Security Scheme, which cover nearly all of the Thai population for the 23 main diseases. ${ }^{12}$ These interventions may have contributed to the better overall outcomes of acute diarrhea. The objectives of this study are to update data on the burdens and patterns of the disease in Thai children under 5 years of age by utilizing the most reliable data available from the Thailand National Health Coverage (NHC) data.

\section{Methods}

Data regarding the admission of acute diarrhea cases by Thai children under 5 years of age with National Health Coverage (NHC). A timeframe between the fiscal years of 2015 and 2019 was used in the study. The authors analyzed inpatient data that represented at least moderately severe acute diarrheal cases. Information was extracted from the summary discharge of all hospitals in Thailand based on NHC data by using the International Statistical Classification of Diseases and Related Health Problems, tenth Revision, Thai Modification (ICD-10-TM). "Acute diarrhea" is characterized by a decrease in stool consistency, loose or liquid stool texture and/or an increase in the frequency of bowel movements to three or more in 1 day. The duration of the disease is classified as acute onset, lasting less than 7 days; prolonged diarrhea, lasting 8 to 13 days; and chronic or persistent diarrhea, lasting 14 days or more. ${ }^{13}$ Acute diarrheal diseases are defined by ICD-10-TM as intestinal infectious disease, A00-A09. Based on these codes, two were categorized. First, the nonspecific diagnosis of infectious disease, i.e. A04: other bacterial intestinal infection; A05: other bacterial foodborne intoxications, not elsewhere classified; A08: viral and other specified intestinal infections; and A09: other gastroenteritis and colitis of infectious and unspecified origin. Last, the more specific diagnosis of infectious diseases included A00: Cholera, A01: Typhoid, A02: other Salmonella infections, A03 Shigellosis, A06: Amoebiasis, and A07: other protozoal intestinal diseases. A02 and A03 were subcategorized to be defined as dysenteric diarrhea. The burden of the disease was based on morbidity, mortality, length of hospital stay (LOS), and cost of medical treatment per admission. The monthly admission rate was assessed to reveal the variation according to the climate of each season. The three common specific diagnostic diseases in each province were presumed to be regional distributors. Data on basic demographics (age and gender), admission details, 
hospital region, LOS, mortality rate, and cost per admission were collected. The present study was approved by the international review board, Center for Ethics in Human Research, Khon Kaen University, Human Research Ethics Committee (\#HE 641527). Consent was waived by Center for Ethics in Human Research, Khon Kaen University because of no personally identifiable data.

\section{Statistical methods}

Continuous and categorical variables are described as medians (and interquartile ranges), means (and standard deviations), and frequencies (\%). Diarrheal-related morbidity and mortality rates are analyzed per 100,000 population for each age group and divided into 0-1 year, $>1$ to 2 years, $>2$ to 3 years, $>3$ to 4 years, and $>4$ to 5 years of age. This rate is calculated by the Poisson regression test. The monthly incidence rate in each year is presented as seasonal peaks. The highest incidence rate of each causative pathogen per province is represented according to the regional distribution. A P value $<0.05$ is considered statistically significant. The data are analyzed using the Stata software package, version 10 (StataCrop LP) program (Texas, USA).

\section{Results}

For the 5-year study period, a total of 579,674 diarrheal-related admissions, in-patient department (IPD), of Thai children under 5 years of age were recorded, with a median of 102,941 persons/year (range: $65,365.5-173,010$ persons/year), and the trend showed a decrease of $17.3 \%$ compared to that in 2010 (124,403 persons/year). Two-thirds of the IPD patients were infants and toddlers; there were 174,799 cases (30.2\%) and 171,211 cases (29.5\%) in children under 1 year of age and aged between 1 and less than 2 years, respectively, and the admission rate declined with increasing age (Fig. 1). Due to the limitation of causative confirmation tests, most diagnoses were based on clinical symptoms. The two most common causes relating to admission were A09 (83.0\%) and A08 (9.8\%), with medians of 98,030 persons/year (range: 94,205.5-115,865.5 persons/year) and 11,758 persons/year (range: 11,262.513,605.5 persons/year), respectively. Other specific diseases, including A00, A01, A02, A03, and A07, were seen to have relatively lower rates: $0.01 \%, 0.2 \%, 0.6 \%$, and $0.05 \%$, respectively.

\section{Diarrheal-related mortality}

Diarreal-related mortality rate was significantly higher in children aged 1 year or less. In this age group, $64.3 \%$ (126/194) of all deaths or 72.1 deaths per 100,000 admissions occurred in first-year children $(P<0.001)$ in the 5 -year period (Table 1$)$. The number of deaths ranged from 37 to 53 deaths/year; therefore, the median diarrheal-related mortality rate was 0.95 per 100,000 population per year (range: 0.81-1.14 per 100,000 population per year). The highest mortality causes were associated with A09 (Table 2). 
Table 1

Mortality rate by age group of Thai children under 5 years from 2015 to 2019

\begin{tabular}{|llll|}
\hline Age group (years) & Admissions & Mortality, No (\%) & P-value \\
\hline$<1$ year & 174799 & $126(30.2 \%)$ & $<0.001$ \\
\hline 1 to $<2$ years & 171221 & $34(29.5 \%)$ & \\
\hline 2 to $<3$ years & 102941 & $14(17.8 \%)$ & \\
\hline 3 to $<4$ years & 75390 & $11(13.0 \%)$ & \\
\hline 4 to $\leq 5$ years & 55323 & $9(9.0 \%)$ & \\
\hline
\end{tabular}

Table 2

Etiology of diarrheal-related mortality by age group in Thai children under 5 years

\begin{tabular}{|llllll|}
\hline Etiology (ICD-10TM) & \multicolumn{5}{c|}{ Fiscal year } \\
\hline & 2015 & 2016 & 2017 & 2018 & 2019 \\
A02 & 3 & 1 & 1 & 3 & 0 \\
A03 & 2 & 0 & 0 & 1 & 0 \\
A04 & 1 & 0 & 2 & 1 & 1 \\
A06 & 0 & 0 & 0 & 0 & 1 \\
A08 & 2 & 0 & 1 & 1 & 3 \\
A09 & 35 & 30 & 32 & 41 & 32 \\
\hline
\end{tabular}

\section{Diarrheal-related LOS and health care expenditure}

Nearly all (99.8\%) admissions involved a stay in the hospital less than 14 days. The diarrheal-related mortality rate in this group was small compared to the group with an LOS of more than 14 days (Table 3). The median estimated hospital expenditure was 36,981,911 Thai baht/year (range: 33,965,67142,699,295.5 Thai baht/year), or 1,126,559.9 United States dollar (USD)/year, (range: 1,034,677.81,300,725.6 USD/year) (November 15, 2021, US Federal Statistical Release year). 
Table 3

The number of diarrheal-related mortality rate by LOS in fiscal year 2015-2019

\begin{tabular}{|lllll|}
\hline $\begin{array}{l}\text { Fiscal } \\
\text { Year }\end{array}$ & $\begin{array}{l}\text { LOS } \\
\text { (Days) }\end{array}$ & $\begin{array}{l}\text { Number of Admissions } \\
(\%)\end{array}$ & $\begin{array}{l}\text { Number of } \\
\text { deaths }\end{array}$ & $\begin{array}{l}\text { Mortality rate/ } 100000 \\
\text { population }\end{array}$ \\
\hline 2015 & $\leq 14$ & $106066(99.8)$ & 39 & 0.97 \\
\hline 2016 & $\leq 14$ & $182(0.2)$ & 4 & 0.74 \\
\hline 2017 & $\leq 14$ & $125948(99.8)$ & 27 & \\
& $>14$ & $196(0.2)$ & 4 & 0.88 \\
\hline 2018 & $\leq 14$ & $105716(99.8)$ & 34 & 1.31 \\
\hline 2019 & $\leq 14$ & $131704(99.9)$ & 2 & 0.72 \\
\hline & $>14$ & $201(0.1)$ & 44 & \\
\hline
\end{tabular}

\section{Year-round variation}

According to the monthly incidences of diarrheal-related admissions, the bimodal peaks were consistent with this pattern over the 5 years (Fig. 2). The first high peak was observed in the cool climate; it sharply increased in November, reached a maximum in January, and then gradually decreased in February. The final low peak occurred during the early rainy season between May and July. In other months, a lower incidence of admissions was seen all year-round, similar to what was seen in a previous study from $2010 .{ }^{4}$

\section{Geographic distribution}

The three specific common foodborne pathogens, Cholera, Typhoid, and Amoebiasis, had distinctive regional distributions. Cholera, which causes severe watery diarrhea, had a high prevalence in the Bangkok metropolis of central and Chonburi Province in the eastern region. These industrial cities are overcrowded with various nationalities and tourists, and therefore easily transmit fecal-oral pathogens. Chonburi Province is a coastal area with brackish water, which is a great habitat for Vibrio cholera. The incidence of Typhoid was highest in the southernmost part of the country (Narathiwat, Songkhla, and Pattani provinces). Moreover, Amoebiasis also occurred in the southern region. Even if the causes of the 
geographic distribution of each pathogen were warranted, overpopulation and sanitation may be the principal causes of fecal-oral transmitted diseases.

\section{Discussion}

Diarrhea is a major global burden disease among children, largely in the under-5-year-old group. In 2013, diarrheal-related deaths in the under five years old group were estimated by the $\mathrm{WHO}^{3}$ as the second leading cause of mortality, which decreased to being the fourth in 2015, as determined by the GBD 2015 working group. ${ }^{5}$ In Thailand, national health insurance consisted of three main schemes, and the Universal Coverage (UC) scheme, which covers three-quarters of the Thai population, was implemented in 2002. 4,11,14 Hospital-based data gathered from the Bureau of Epidemiology of the Ministry of Public Health of Thailand from 2015 to 2019 were analyzed to estimate the burden and pattern of acute diarrhea in under 5 years old Thai children. Over the last 4 decades, the annual incidence of diarrhea in Thai children under 5 years of age has continuously increased. From 1978 to $1983^{15}$, the average incidence was 1,609 cases per 100,000 population, which gradually increased to $7,140.9$ cases per 100,000 population in 2000 and reached 10,000 cases per 100,000 population in $2010 .{ }^{4}$ In 2015 , GBD 2015 assessed that diarrheal episodes from 2005 to 2015 in Thai children younger than 5 years decreased by half $(-47.3 \%,(-59.1$ to -32.9$))$ and episodes of children younger than 5 years declined by $10.4 \%(9.1-11.6 \%)$ worldwide. $^{5}$ This study also revealed an identical trend - diarrheal-related admissions decreased by approximately 20,000 cases/year or $17.3 \%$ compared to the research results obtained in Thailand in $2010 .{ }^{4}$ Along with the diarrheal-related mortality rate in our country, it continuously declined from 2.0 to 0.7 per 100,000 population in 1978 to $1983^{15}$ and sharply decreased by $70.8 \%$ ( -82.4 to $-51.0 \%$ ) from 2005 to 2015 . On a broader scale, it decreased by $39.2 \%$ among children under 5 years of age in this period. ${ }^{5}$ As in our study, the death rate from diarrhea remained constant at a low rate of approximately 0.95 per 100,000 population per year (range: $0.81-1.14$ per 100,000 population per year) in the 5-year study period. Notably, in our study, an approximately three-fourths diarrheal-related mortality rate occurred in children under 2 years of age, similar to what was seen in previous studies in different periods in both Thailand and other countries. A published investigation from Thailand over a 1-year period from 1985 to $1986^{16}$ studied children under 5 years of age with acute diarrhea. Half of them were younger than 1 year old, and $84 \%$ were younger than 2 years old. In 2006 , Wilunda et al. ${ }^{17}$ studied factors associated with diarrhea in Thai children under 5 years of age in 2006 and concluded that the age group between 6 and 23 months of age was the high-risk group for diarrheal disease. Consistent with studies in Asia (Federal Democratic Republic of Nepal) and Africa (Arab Republic of Egypt and Federal Democratic Republic of Ethiopia), this age group is seen to have a high risk of diarrhea, which may be affected by immature intestinal immunity to enteropathogens and environmental exposure, and may be related to the introduction of complementary food. ${ }^{7,17-18}$ Nepal, ref Ethiopia Moreover, the diarrheal rates among the group of children under 5 years of age with LOS less than 14 days were lower than those of children with

LOS longer than 14 days, which is consistent with previous studies. ${ }^{4,15}$ In Thailand, there are three factors that contributed to the lower rate of diarrheal death, of which the first is the achievement of national 
insurance, especially the UC scheme in 2002, that covers nearly the entire Thai population. Second, there are national and accessible oral rehydration therapies, such as those including ORSs. Last, the implementation of a rotavirus vaccine (RV) as an optional vaccine in 2012 and was added to the Expanded Program of Immunization (EPI) in 2020. All these factors may have had a positive impact on the incidence and mortality rate among Thai children. The constant pattern of seasonal variation demonstrated consistency over the 5-year period. The higher peak in winter (November to February) was followed by a smaller peak in the early rainy season (May to July), similar to prior data obtained in 2010. The peak during the cool weather period might be from rotavirus outbreaks in dry and cool conditions in which microorganisms tend to peak, also called winter diarrhea. Previous literature reviewed the seasonal effect on the incidence of acute diarrhea in Northeast Thailand from 1982 to 1987 and reported that the apex of the incidence was inversely related to an abrupt decrease in the temperature in the first month of each year. ${ }^{20}$ While the minor peak could be from dysentery or rainy-season diarrhea, this bacterial diarrhea peaked in hot, dry, and early rainy climates. 4,10,15,21-25 The peak winter diarrhea decreased with age, while the rainy-season diarrheal peak increased with advanced age. ${ }^{20}$ In contrast with a study from the Federal Democratic Republic of Nepal ${ }^{18}$, their results indicated a unique seasonal difference, which was a risk of incidence of diarrheal-related admission in spring and summer and a lower risk of disease in autumn and winter. They hypothesized that it was caused by bacteria because the peaks usually occurred in hotter and rainier months. ${ }^{26}$ This may be the result of different topographies and climates determining the effect of spreading pathogens. The high density of patients in each geographical area with Cholera, Typhoid, and Amoebiasis may be explained by the environment being suited for the growth of pathogens. In the Bangkok metropolis, the population with the highest density could more easily transmit causative pathogens via the fecal-oral route. The other coastal provinces were not only crowded by tourism but also rich in seafood, which was a great source of nontyphoidal and Vibrio species. Due to the limitation that current practice does not recommend identifying all causative pathogens causing acute diarrhea in all patients ${ }^{12}$, our data were obtained from the ICD-10 TM, and the diagnosis was based on clinical presentation. Nearly all of the hospitalized diarrhea patients were due to A08 and A09. In Thailand, data from other studies revealed that rotavirus was the major infectious agent causing watery diarrhea and leading to death in children younger than 5 years. ${ }^{27-29}$ Rotavirus vaccines have been licensed as optional vaccines in Thailand since 2012 and incorporated in the Expanded Program of Immunization (EPI) in 2020. The effectiveness of RV against acute diarrhea in children under 5 years of age before and after incorporation of RV into the EPI is warranted. However, our study has some limitations. First, this study was retrospective; consequently, the data and results may be inaccurate due to incomplete medical records or ICD-10 TM codes. Moreover, the current practical management and clinical practice guidelines for acute gastroenteritis do not recommend identifying the pathogens in all patients, which might lead to miscoding or misclassification.

\section{Conclusions}

The burden, incidence and mortality rate of acute diarrhea in Thai children under five years of age were lower than those in the past, but the cost of treatment is still high. The most common cause contributing 
to admission was A09. A similar seasonal outbreak of acute diarrhea was observed each year. The causative agent was not significant and was mainly unspecific. The effectiveness of RV against acute diarrhea in children under 5 years of age is supported.

\section{Abbreviations}

NHC: National Health Coverage; ICD-10-TM: International Statistical Classification of Diseases and Related Health Problems, tenth Revision, Thai Modification; WHO: World Health Organization; UNICEF: United Nations Children's Fund; GBD: Global Burden of Diseases, Injuries, and Risk factor Study; ORS: oral rehydration salts; UC: Universal Coverage Scheme; CSMBS: Civil Servant Medical Benefit Scheme; LOS: length of hospital stay; IPD: in-patient department; USD: United States dollar; RV: rotavirus vaccine; EPI: Expanded Program of Immunization.

\section{Declarations}

\section{Ethics approval and consent to participate}

The present study was approved by the international review board, Center for Ethics in Human Research, Khon Kaen University, Human Research Ethics Committee (\#HE 641527). Consent was waived by Center for Ethics in Human Research, Khon Kaen University because of no personally identifiable data. The authors confirm that all methods were performed in accordance with the relevant guidelines and regulations."

\section{Consent for publication}

Not applicable

\section{Availability of data and materials}

The data that support the findings of this study are available from Thailand National Health Coverage but restrictions apply to the availability of these data, which were used under license for the current study, and so are not publicly available. Data are however available from the authors upon reasonable request and with permission of Thailand National Health Coverage

\section{Competing interests}

The authors declare that they have no competing interests.

\section{Funding}




\section{Authors' Information}

${ }^{1}$ Department of Pediatrics, Srinagarind Hospital, Faculty of Medicine, Khon Kaen University, Thailand

${ }^{2}$ Clinical Epidemiology unit, Srinagarind Hospital, Faculty of Medicine, Khon Kaen University, Khon Kaen, Thailand

\section{Contributions}

BC conceptualized and designed the study, performed the computations, discussed the results, drafted the initial manuscript and contributed to the final manuscript. KS, performed the computations, involve in data acquisition, discussed the results, drafted the initial manuscript and contributed to the final manuscript. WP performed the computations, discussed the results, contributed to the final manuscript. NL performed the computations, discussed the results, contributed to the final manuscript. SS performed the computations, involve in data acquisition, discussed the results, contributed to the final manuscript. KT performed the computations, involve in data acquisition, responsible for analysis and interpretation of data, designed the figures and tables, contributed to the final manuscript. All authors review and approved the final manuscript.

\section{Corresponding author}

Correspondence to: Busara Charoenwat, MD

Department of Pediatrics, Srinagarind Hospital, Faculty of Medicine, Khon Kaen University, Thailand ORCID number: 0000-0003-4892-4993

\section{Acknowledgements}

The authors are grateful to the patients, The Royal College of Pediatricians of Thailand, Pediatric Society of Thailand, staff of the Srinagarind Hospital, Faculty of Medicine, Khon Kaen University. English language and style in this article was provided by qualified editor from American Journal Editing service (AJE).

\section{References}

1. World Health Organization.Diarrhoeal disease.https://www.who.int/news-room/factsheets/detail/diarrhoeal-disease. Accessd 14 Dec 2021. 
2. Guarino A, Lo Vecchio A, Dias JA, Berkley JA, Boey C, Bruzzese D, et al.Universal Recommendations for the Management of Acute Diarrhea in Nonmalnourished Children.J Pediatr Gastroenterol Nutr.2018;67(5):586-93.

3. Farthing M, Salam MA, Lindberg G, Dite P, Khalif I, Salazar-Lindo E, et al.Acute diarrhea in adults and children: a global perspective.J Clin Gastroenterol.2013;47(1):12-20.

4. Sutra S, Kosuwon P, Chirawatkul A, Thepsuthammarat K.Burden of acute, persistent and chronic diarrhea, Thailand, 2010.J Med Assoc Thail.2012;95 Suppl 7:S97-107.

5. GBD Diarrhoeal Diseases Collaborators.Estimates of global, regional, and national morbidity, mortality, and aetiologies of diarrhoeal diseases: a systematic analysis for the Global Burden of Disease Study 2015.Lancet Infect Dis.2017;17(9):909-48.

6. OCHA Services.Ending Preventable Child Deaths from Pneumonia and Diarrhoea by 2025:The integrated Global Action Plan for Pneumonia and Diarrhoea (GAPPD).https://reliefweb.int/report/world/ending-preventable-child-deaths-pneumonia-anddiarrhoea-2025. Accessd 14 Dec 2021.

7. Qazi S, Aboubaker S, MacLean R, Fontaine O, Mantel C, Goodman T, et al.Ending preventable child deaths from pneumonia and diarrhoea by 2025. Development of the integrated Global Action Plan for the Prevention and Control of Pneumonia and Diarrhoea.Arch Dis Child.2015;100 Suppl 1:S23-28.

8. Mohammed S, Tamiru D.The Burden of Diarrheal Diseases among Children under Five Years of Age in Arba Minch District, Southern Ethiopia, and Associated Risk Factors: A Cross-Sectional Study.Int Sch Res Not.2014;2014:1-6.

9. Aziz FAA, Ahmad NA, Razak MAA, Omar M, Kasim NM, Yusof M, et al.Prevalence of and factors associated with diarrhoeal diseases among children under five in Malaysia: a cross-sectional study 2016.BMC Public Health.2018;18(1):1363.

10. Global Burden of Disease Study 2013 Collaborators.Global, regional, and national incidence, prevalence, and years lived with disability for 301 acute and chronic diseases and injuries in 188 countries, 1990-2013:a systematic analysis for the Global Burden of Disease Study 2013.Lancet Lond Engl.2015;386(9995):743-800.

11. Paek SC, Meemon N, Wan TTH.Thailand's universal coverage scheme and its impact on healthseeking behavior.SpringerPlus.2016;5(1):1952.

12. Treeprasertsuk S, Thepsuthammarat K, Kitsahawong B, Phaosawasdi K.Acute diarrhea, a significant burden to Thailand's universal health care system: a nationwide database.Asian Biomed.2017;10(s1):s23-30.

13. Guarino A, Ashkenazi S, Gendrel D, Lo Vecchio A, Shamir R, Szajewska H, et al.European Society for Pediatric Gastroenterology, Hepatology, and Nutrition/European Society for Pediatric Infectious Diseases evidence-based guidelines for the management of acute gastroenteritis in children in Europe: update 2014.J Pediatr Gastroenterol Nutr.2014;59(1):132-52.

14. Sumriddetchkajorn K, Shimazaki K, Ono T, Kusaba T, Sato K, Kobayashi N.Universal health coverage and primary care, Thailand.Bull World Health Organ.2019;97(6):415-22. 
15. Phonboon K, Kunasol P, Chayaniyayodhin T, Srisomporn D.Surveillance of diarrhoeal diseases in Thailand.Bull World Health Organ.1986;64(5):715-20.

16. Echeverria P, Taylor DN, Lexsomboon U, Bhaibulaya M, Blacklow NR, Tamura K, et al.Case-control study of endemic diarrheal disease in Thai children.J Infect Dis.1989;159(3):543-8.

17. Wilunda C, Panza A.Factors Associated with Diarrhea among Children Less Than 5 Years Old in Thailand: A Secondary Analysis of Thailand Multiple Indicator Cluster Survey 2006.J Health Res.2009;23(Suppl.):17-22.

18. Li R, Lai Y, Feng C, Dev R, Wang Y, Hao Y.Diarrhea in under Five Year-Old Children in Nepal: A Spatiotemporal Analysis Based on Demographic and Health Survey Data.Int J Environ Res Public Health.2020;17(6):2140.

19. El-Gilany AH, Hammad S.Epidemiology of diarrhoeal diseases among children under age 5 years in Dakahlia, Egypt.East Mediterr Health J.2005;11(4):762-75.

20. Pinfold JV, Horan NJ, Mara DD.Seasonal effects on the reported incidence of acute diarrhoeal disease in northeast Thailand.Int J Epidemiol.1991;20(3):777-86.

21. Jiraphongsa C, Bresee JS, Pongsuwanna Y, Kluabwang P, Poonawagul U, Arporntip P, et al.Epidemiology and burden of rotavirus diarrhea in Thailand: results of sentinel surveillance.J Infect Dis.2005;192 Suppl 1:S87-93.

22. Echeverria P, Seriwatana J, Taylor DN, Yanggratoke S, Tirapat C.A comparative study of enterotoxigenic Escherichia coli, Shigella, Aeromonas, and Vibrio as etiologies of diarrhea in northeastern Thailand.Am J Trop Med Hyg.1985;34(3):547-54.

23. Kamath KR, Feldman RA, Rao PS, Webb JK.Infection and disease in a group of South India families. II. General morbidity patterns in families and family members.Am J Epidemiol.1969;89(4):375-83

24. Echeverria P, Seriwatana J, Taylor DN, Tirapat C, Chaicumpa W, Rowe B.Identification by DNA hybridization of enterotoxigenic Escherichia coli in a longitudinal study of villages in Thailand.J Infect Dis.1985;151(1):124-30.

25. Echeverria P, Harrison BA, Tirapat C, McFarland A.Flies as a source of enteric pathogens in a rural village in Thailand.Appl Environ Microbiol.1983;46(1):32-6.

26. Chao DL, Roose A, Roh M, Kotloff KL, Proctor JL.The seasonality of diarrheal pathogens: A retrospective study of seven sites over three years.PLoS Negl Trop Dis.2019;13(8):e0007211.

27. Maneekarn N, Khamrin P.Rotavirus associated gastroenteritis in Thailand.Virusdisease.2014;25(2):201-7.

28. Tharmaphornpilas $P$, Jiamsiri S, Boonchaiya S, Rochanathimoke $O$, Thinyounyong W, Tuntiwitayapun $S$, et al.Evaluating the first introduction of rotavirus vaccine in Thailand: Moving from evidence to policy.Vaccine.2017;35(5):796-801.

29. Chieochansin T, Vutithanachot V, Phumpholsup T, Posuwan N, Theamboonlers A, Poovorawan Y.The prevalence and genotype diversity of Human Rotavirus A circulating in Thailand, 2011-2014.Infect Genet Evol.2016;37:129-36. 
Figures

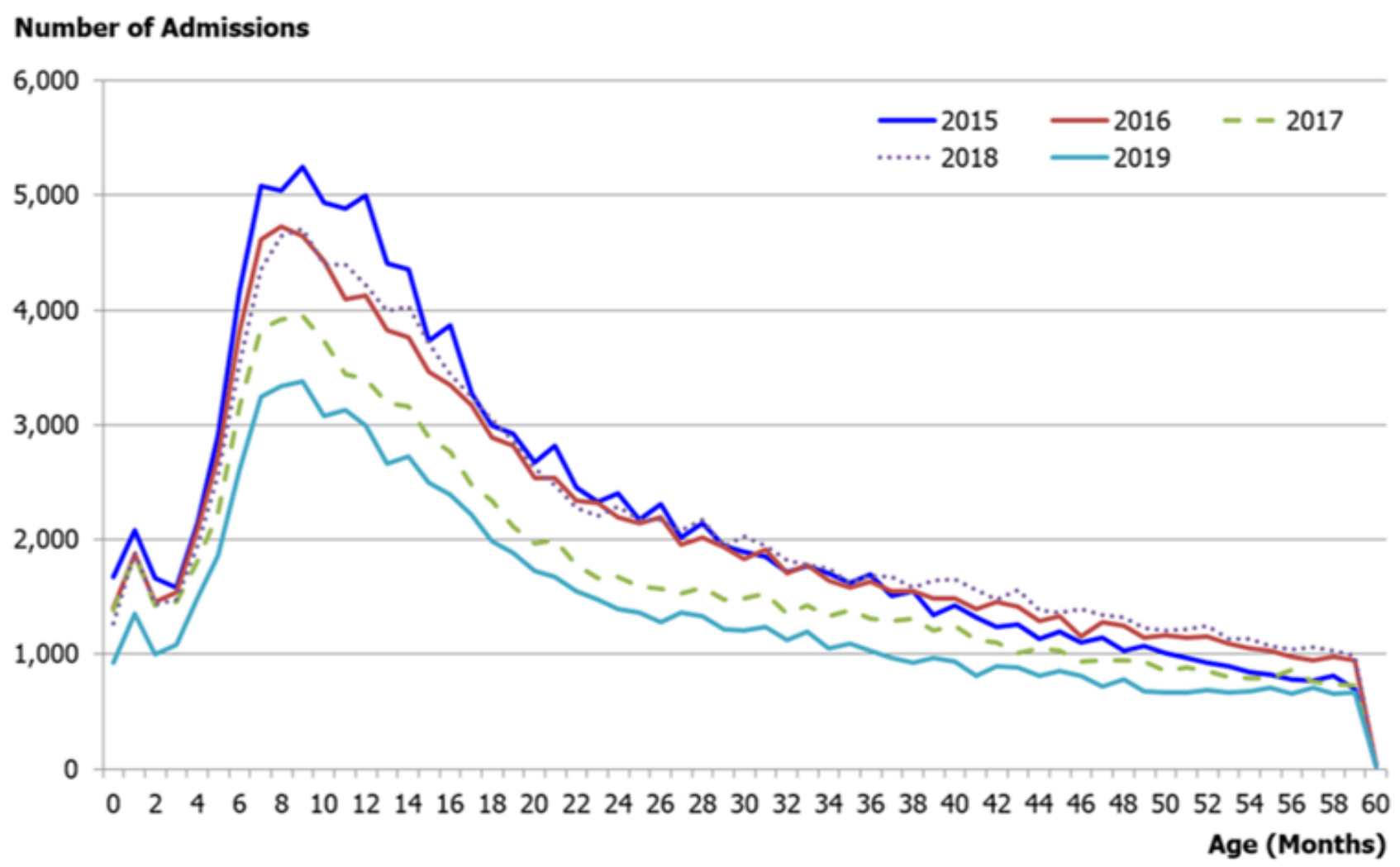

Figure 1

Diarrheal-related admissions in Thai children under 5 years by age (month) from 2015 to 2019 


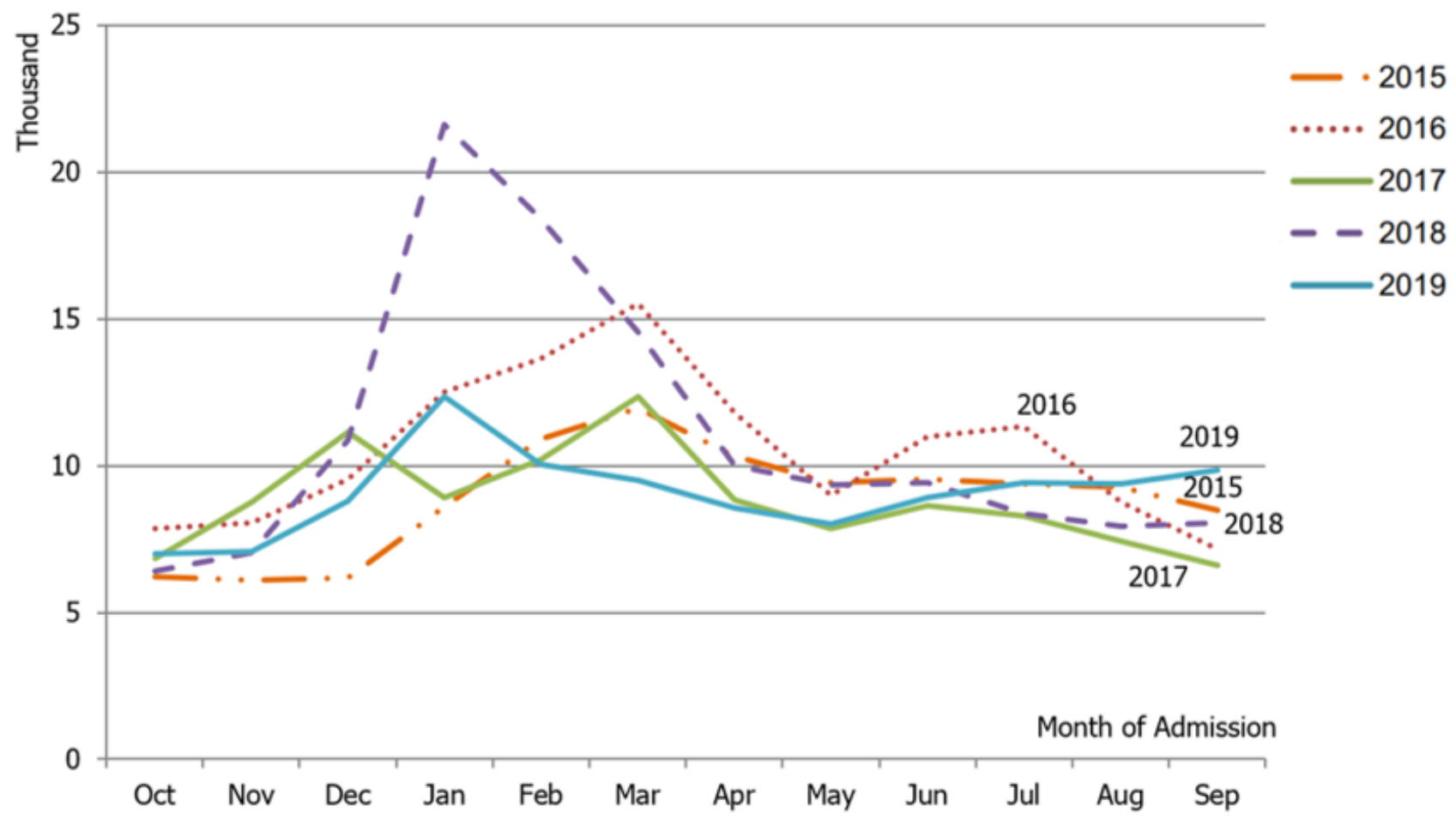

Figure 2

The number of monthly admissions per 1,000 Thai children under 5 years by age 